\title{
Addiction: A highly successful, essentially contested concept
}

\author{
Introduction
}

Within two or three decades an increasing use of the concept of addiction has been observed in the media, in expert discourses, in popular culture, and in everyday talk. The concept does not only cover traditional substance use but also a growing range of behaviors like gambling, internet use, sex, eating, work, and shopping. One important reason behind the concept's "use value" lies most likely in the fact that it can be adapted to so many different situations of contemporary life. The concept also integrates everyday talk with medical and scientific discourse (Room, Hellman, \& Stenius, 2015; Valverde, 1998). The scientific sector is supposed to produce the most reliable, transparent, objective, and impersonal knowledge (Warshal, 2010), and as such the concept holds the credibility label of being "made by science."

However, despite its widespread use, the term "addiction" carries a crucial controversy that troubles the criteria of scientific knowledge. The controversy concerns whether such a general concept, one that implies a unitary and universal structure in different behaviors, should be justified in the first place, and if it should, what kind of implications are involved in the conceptualization of certain ways of behaving, acting, or choosing as "addicted." These criteria and implications have become a concern of research on both history of science and contemporary scientific practices, as well as concept analytical research shedding light on the moral and normative underpinnings of the scientific discourses on addiction. This is important because the understandings of the concept in the expert discourses, such as the discourse of medical practitioners, also shape the conditions of those living through these issues every day.

By virtue of being highly controversial yet frequently used in all parts of society, the concept of addiction has many similarities with what Sulkunen in this issue calls "essentially contested concepts." The term "essentially contested concept" was introduced by Walter Bryce Gallie (1956a, 1956b, 1964) to facilitate an understanding of the different interpretations and use of multivalent terms such as "art" and "social justice." According to Garver (1978, p. 168 ), the term essentially contested concept gives a name to a problematic situation where a variety of meanings can be employed for key terms in an argument, and where these different meanings can or should not be meet with dogmatism ("My answer is right and all others are wrong"), skepticism ("All answers are equally true (or false); everyone has a right to his own truth"), or eclecticism ("Each meaning gives a partial view so the more meanings the better").

This special issue addresses some of the central controversies of the addiction concept, with a starting point that the concept of addiction is formed within, and varies between, different expert (mostly scientific) discourses. Recently, following the fast developments in neuroscience, research on neuroscientific causes, processes, and mechanisms has to some degree been favored at the expense of psychosocial models of addictive behaviors. However, it is increasingly recognized that in order to complete the great puzzle of risk consumption, more effort should be put on environmental explanations (Kalant, 2015) and the interactions between different fields of knowledge. If there is a common structure in which all types of risk consumption is rooted, this might not be located in particular biological, psychosocial, or cultural processes, but in the interaction between them.

\section{Key Controversies}

In this introduction, we want to highlight three subjects for discussion that illustrate certain key controversies on the concept of addiction: its definition, range of use, and the value it may have for both academic production of knowledge and practical situations. These controversies are related to the question of addiction in specific ways, but are also influencing how risk consumption is understood and handled in society in general. These controversies can also be found among the initial sparks for the conference "Addiction: What is the added value of the concept today?" held at Hotel Majvik, Helsinki, Finland, on October 14-17, 2012.

One topic of dispute regards whether we need one or many concepts to cover the variety of different risk behaviors associated with addiction today. As Sulkunen (2015) points out, research, but also practice, needs the concept of addiction in order to structure knowledge and compare between different instances of addictive behavior. The patients need the concept when they want to understand why they act in a certain way. Sulkunen provides tools for approaching the structuring of addictive behavior in both scientific and lay discourse through an images theory of addiction. Simon and West (2015) give an overview of different models of addiction that may serve as a theoretical framework on which further development of prevention 
and treatment may be based. Although the models represent different approaches to the concept of addiction, they all talk about addiction as a repeated, powerful motivation to engage in a particular behavior despite the experience of risk and significant harm. Ylikoski and Pöyhönen (2015) make addiction into a test case for improving the models of diagnostic criteria. They propose that addiction could be seen as a kind with certain core elements situated in a matrix of causal relations, which would make addiction recognizable despite its many variations. Although the authors refuse an essentialist idea of a common core, they aim at one model of addition in order to facilitate theory building, whereas it will be up to empirical studies to confirm the existence of this kind.

Several articles in the special issue show how the modern concept of addiction and the epistemological underpinnings into which it is tied varies not only in historically and culturally but also in one and the same culture within a particular period of time. Room et al. (2015) track the history of the general conceptual structure of addiction as an inner compulsion and differentiate it from several other terms describing excessive behaviors. They follow Harry Levine's (1978) groundbreaking notion on how the concept of addiction as uncontrollable compulsion became a part of 19th-century medical thought and point out that Levine did not differentiate between the concept and the diverse terms. The terms can be found much earlier, and their variation is also a concern of the contemporary attempts for defining these problems. The genealogy of the addiction concept also has often posited addiction as a problem for the individual, but Petrilli and Beccaria (2015) show how, in the Italian scientific community of the late 1800s and early 1900s, alcoholism—Levine's test case-was not defined only as a problem of the individual but also as a social problem.

Another issue regards whether or how addition is rooted in biological, psychosocial, or cultural conditions. The dominance of psychosocial models in the late 20th century was challenged and to some extent replaced by neuroscientific approaches. However, as indicated by Helén and Toivio (2015), this process was not necessarily the result of competition where the neuroscientific approaches outdid the psychosocial. Rather, the relationship between the neuroscientists and psychosocial therapeutic systems can be seen as a reciprocal exchange, where the neuroscientist research milieus use the therapeutic system to legitimize their research, whereas the therapeutic system uses neuroscientific results in therapy.

In this happy companionship between psychologists and neuroscientists, social scientists and humanistic researchers seem to have problems legitimating their approaches. However, as pointed by Kalant (2015) from the perspective of neurobiology and Simmat-Durand and Koski-Jännes (2015) from the perspective of social sciences, research strongly indicates that in the group of psychiatric disorders, addiction may be the very case in point where the interactions between biologic, psychosocial, and cultural explanations need to be taken into account in their full force in both research as well as prevention and harm- reducing work. As noted by Kalant, addictive disorders are understood in certain ways depending on the dominant scientific presuppositions, which may change if they are tested properly. As noted by Simmat-Durand and KoskiJännes, there are remarkable differences as to how substance-use disorders are defined and understood by treatment professionals in different countries.

The third controversy regards the addict's degree of agency. Neuroscience has found decision-making capacities to be damaged in persons suffering from addictive disorders, and the brain disease concept of addiction advances the idea that addicts cannot be held responsible for their behavior. According to Borch (2015), pathological gamblers do not know and cannot give a reason why they keep on gambling even though they know how damaging it is. Borch's results imply that pathological gambling is unintentional and hence that the gamblers cannot be blamed for their risk consumption. Addiction can also be seen as a particular locus for the paradoxes concerning the intentional choice to act self-destructively. The question of akrasia, defined as acting against one's better judgment, can be seen as a traditional philosophical question that can be illustrated through addictive behavior, and the inability to name a motivation to it as one of its central traits. Drawing on Donald Davidson's views on akrasia, Heather and Segal (2015) argue that it is possible to interpret addictive behavior within the language of intentional agency. Acting like this is not necessarily paradoxical, but it cannot be held rational. The absence of rational motivation, they conclude, may also explain the inability of addict to name a motivation to their behavior. According to Uusitalo (2015), and in contrast to the strand of thought of addiction as a disease that damages a person's capacity for judgment and thus impairs responsibility, it is important to recognize that addicts are morally accountable for their addictive action. This is, amongst other reasons, because research indicates that seeing addicts as individuals with full-blown human agency responsible for their choices is proven to increase the effect of treatment as the addicts feel that they have control over their actions and are respected. Addicts should, however, not be blamed for their actions, as this might be counteractive in therapeutic work.

\section{The added value of the concept today}

Despite many controversies, the concept of addiction is referred to increasingly often and in new contexts. The primary example of this is, of course, the addition of the term "addictive disorders," including gambling, in the American Psychiatric Association's Fifth Edition of the Diagnostic and Statistical Manual of Mental Disorders in 2013. One important factor behind the concept's success is that it offers scientifically approved theories explaining what risk consumption is and, hence, how it can be handled. Other added values are the cultural images of addiction and the addict that not only influence and are influenced by how risk consumption and groups are perceived and handled in society, but also influence and are influenced by what it means to be a man and a woman, adult and child, rich and poor, and what it means to live in a welfare society today. Addiction is thereby more than a 
concept explaining risk behavior and an object of scientific inquiry; it is also a practice. It is a practice of forming academic knowledge and treatment programs, and it is a practice embedded in everyday lives. In the end, the concept of addiction addresses matters of life itself: the vulnerabilities, recoveries, and endings of life.

If there is a need for such a concept, is the concept of addiction the only concept that can cover this need? The greatest criteria of success of the concept of addiction might be that it resonates to a need in society for a flexible, essentially contested concept that covers a variety of meanings regarding interpretation and use. As such we can argue that even though the understandings of risk behaviors share many of the same characteristics, there are many concepts of addition that may operate simultaneously but dominate in a certain period, and that empirical research on addiction-related issues has always, conditioned by these underpinnings, resonated with medical and social practices. Indeed, an unambiguous concept might make it easier to agree how the risk questions shall be understood and handled. However, it may be that excessive behaviors do not follow any general template that could be empirically grounded. Acting as if they do runs the risk that the problems are handled in the wrong way.

One the one hand, some theory is necessary to describe risk consumption. Amongst other things, patients need explanations of their behaviors that they do not understand, as well as help in their efforts to change. On the other hand, we will warn against a notion suggesting that it is utterly irrelevant what kind of concepts and words we are using to describe such phenomena as risk consumption. As pointed out by Borch in this issue, the concept of addiction is highly political, as it binds the knowledge of risk consumption to the psychiatric and (increasingly) the neuroscientific field of knowledge, which again is highly determined for what kind of knowledge which will be produced, what kind of expertise which will be developed, and how the problems will be understood and dealt with.

We started this introduction by emphasizing the addiction concept's great success in terms of constantly invading new sectors and types of risk consumption. In the same style we will close this introduction by suggesting that the concept's increasing success might be its greatest danger, as words expanding from "nothing" to "everything" may end up meaning "nothing." If one concept dies, a new and even more convincing concept may arise. If that happens, we hope that this concept will not only focus on biological and psychosocial causes, processes, and mechanisms, or only on biological, psychosocial, or cultural explanations separately, but also focus on the interaction between them. For only by setting aside vested interests and approaching the risk consumer wholly as brain, person, and culture, the great puzzle of risk consumption can be completed.

\section{References}

American Psychiatric Association. (2013). Diagnostic and statistical manual of mental disorders (5th ed.).
Arlington, VA, United States: American Psychiatric Publishing.

Borch, A. (2015). Problem gambling: A Lacanian real. International Journal of Drug and Alcohol Research, 4(1), 71-76.

Gallie, W. B. (1956a). Essentially contested concepts. Proceedings of the Aristotelian Society, 56, 167-198.

Gallie, W. B. (1956b). Art as an essentially contested concept. The Philosophical Quarterly, 6(23), 97-114.

Gallie, W. B. (1964). Essentially contested concepts. In W. B. Gallie (Ed.), Philosophy and the historical understanding, (pp. 157-191). London, England: Chatto \& Windus.

Garver, E. (1978). Rhetoric and essentially contested arguments. Philosophy and Rhetoric, 11(3), 156-172.

Heather, N., \& Segal, T. (2015). Is addiction a myth? Donald Davidson solution to the problem of akrasia says not. International Journal of Drug and Alcohol Research, 4(1), 77-83.

Helén, I., \& Toivio, J. (2015). The brain craving for gambling? Neurosciences and addiction concept in clinical practice. International Journal of Drug and Alcohol Research, 4(1), 45-51.

Kalant, H. (2015). Neurobiological research on addiction: What value has it added to the concept? International Journal of Drug and Alcohol Research, 4(1), 53-59.

Levine, H. G. (1978). The discovery of addiction: Changing conceptions of habitual drunkenness in America. Journal of Studies on Alcohol, 39, 143-174.

Petrilli, E., \& Beccaria, F. (2015). The Italian "alcohol question” from 1860 to 1930: Two opposing scientific interpretations. International Journal of Drug and Alcohol Research, 4(1), 37-43.

Room, R., Hellman, M. \& Stenius, K. (2015). Addiction: The dance between concept and terms. International Journal of Drug and Alcohol Research, 4(1), 27-35.

Simmat-Durand, L., \& Koski-Jännes, A. (2015). Cannabis and other illicit drugs: Beliefs among French and Finnish addiction treatment providers. International Journal of Drug and Alcohol Research, 4(1), 61-69.

Simon, R., \& West, R. (2015). Models of addiction and types of interventions: An integrative look. International Journal of Drug and Alcohol Research, 4(1), 13-20.

Sulkunen, P. (2015). The images theory of addiction. International Journal of Drug and Alcohol Research, 4(1), 5-11.

Uusitalo, S. (2015). Addiction, recovery, and moral agency: Philosophical considerations. International Journal of Drug and Alcohol Research, 4(1), 85-89.

Valverde, M. (1998). Diseases of the will: Alcohol and the dilemmas of freedom. Cambridge, England: Cambridge University Press.

Warshal, K. (2010). Molding interpersonal relation through conditional clauses: Consensus-building strategies in written academic discourse. Journal of English Academic Purposes, 9, 140-150.

Ylikoski, P. \& Pöyhönen, S. (2015). Addiction-as-a-kind hypothesis. International Journal of Drug and Alcohol Research, 4(1), 21-25. 
4 Anita Borch and Varpu Rantala

Anita Borch, Ph.D.

National Institute for Consumer Research (SIFO)

Oslo, Norway

Email: anita.borch@sifo.no

Varpu Rantala, Doctoral Student

Media Studies, University of Turku, Finland

Associate Partner in Centre for Research on Addiction,

Control and Governance (CEACG), Helsinki, Finland

Email: varran@utu.fi 